EUROPEAN JOURNAL OF PURE AND APPLIED MATHEMATICS

Vol. 14, No. 4, 2021, 1350-1366

ISSN 1307-5543 - ejpam.com

Published by New York Business Global

\title{
Coincidence and Common Fixed Point Theorems in $G_{b}$-Cone Metric Spaces
}

\author{
Saadia Benchabane ${ }^{1, *}$, Smaïl Djebali ${ }^{1,2}$ \\ 1 Laboratoire "Théorie du Point Fixe et Applications", ENS, BP 92 Kouba. Algiers, 16006. \\ Algeria \\ 2 Department of Mathematics 6 Statistics, College of Sciences, Imam Mohammad Ibn Saud \\ Islamic University (IMSIU), PB 90950. Riyadh 11623, Saudi Arabia
}

\begin{abstract}
In this article, some coincidence and common fixed point results were obtained for four mappings satisfying some special contractive conditions and defined on a $G_{b}$-cone metric space (with or without the assumption of normality). Our results generalize recent results in the literature. Two illustrative examples are included and some consequences are provided.
\end{abstract}

2020 Mathematics Subject Classifications: 47H10, 54E50, 54H25

Key Words and Phrases: Coincidence and common fixed point, $G_{b}$-Cone Metric Spaces.

\section{Introduction and preliminaries}

The notion of cone metric space is an important generalization of the classical concept of metric. It was introduced in 2007 by Huang and Zhang [18] who extended the Banach contraction principle in the setting of cone metric spaces. Their result has been generalized in several directions by many authors (see, e.g., [14], [8], [5], [1], [4], [12], [17]) and there has been a number of generalizations of the notion of a cone metric space. One such generalization is that of the $G$-cone metric space initiated by Ismat Beg et al. [10]. Recently, Ughade and Daheriya [3] introduced the concept of $G_{b}$-cone metric space as a generalization of $G$-cone metric space and obtained some fixed point (and common fixed point) results.

In this paper, our aim is to establish some coincidence and common fixed point results for four mappings, defined on a $G_{b}$-cone metric space without the assumption of normality and also with the assumption of normality. The mappings satisfy some special contractive conditions. We first collect some basic notions and primary results needed to develop our existence results. $\mathbb{N}$ will refer to the set of nonnegative integers. Our main existence results are Theorem 1 (without the assumption of normality) and Theorem 2 (with the assumption of normality). In each case, an example of application and a corollary are supplied.

*Corresponding author.

DOI: https://doi.org/10.29020/nybg.ejpam.v14i4.4126

Email addresses: benchabane.saadia@ymail.com (S. Benchabane), djebali@hotmail.com (S. Djebali) 
Definition 1. Let $E$ be a real Banach space with norm $\|\cdot\|$ and $P$ a subset of $E$. Then $P$ is called a cone if and only if

(i) $P$ is closed, nonempty, and $P \neq\{\theta\}$, where $\theta$ is the zero vector in $E$,

(ii) if $a, b \geq 0$, and $x, y \in P$, then $a x+b y \in P$,

(iii) if $x \in P$ and $-x \in P$, then $x=\theta$.

Given a cone $P$ in a Banach space $E$, a partial ordering $\preceq$ with respect to $P$ is given by

$$
x \preceq y \text { if and only if } y-x \in P .
$$

We write $x \prec y$ whenever $x \preceq y$ and $x \neq y$, while $x \ll y$ will stand for $y-x \in \operatorname{int}(P)$, where $\operatorname{int}(P)$ designates the interior of $P$. If $\operatorname{int}(P) \neq \emptyset$, then $P$ is called a solid cone. The cone $P$ is called normal if there is a number $k>0$, such that for all $x, y \in E$, we have

$$
\theta \preceq x \preceq y \text { implies }\|x\| \leq k\|y\| \text {. }
$$

The least positive number satisfying this inequality is called the normal constant of $P$. In [6], it is proved that there are no normal cones with a normal constant $k<1$.

Lemma 1. [13] For cones which are not normal, the following properties hold

(PT1) If $u \preceq v$ and $v \ll w$, then $u \ll w$.

(PT2) If $u \ll v$ and $v \preceq w$, then $u \ll w$.

(PT3) If $u \ll v$ and $v \ll w$, then $u \ll w$.

(PT4) If $\theta \preceq u \ll c$ for each $c \in$ int $P$, then $u=\theta$.

(PT5) If $a \preceq b+c$ for each $c \in$ int $P$, then $a \preceq b$.

(PT6) If $E$ be a real Banach space with a cone $P$, and if $a \preceq \lambda a$, where $a \in P$ and $0 \leq \lambda<1$, then $a=\theta$.

(PT7) If $c \in$ int $P, a_{n} \in E$ and $a_{n} \rightarrow \theta$, then there exists an $n_{0} \in \mathbb{N}$ such that, for all $n>n_{0}, a_{n} \ll c$.

Definition 2. [3] Let $X$ be a nonempty set and $E$ a real Banach space equipped with the partial ordering $\preceq$ with respect to the cone $P$. A vector-valued function $G: X \times X \times X \rightarrow X$ is said to be a generalized cone $b$-metric function on $X$ with the constant $s \geq 1$ if the following conditions are satisfied

(GBC1) $G(x, y, z)=\theta$ if $x=y=z$,

(GBC2) $\theta \prec G(x, y, z)$, for $y \neq z$, for all $x, y, z \in X$,

(GBC3) $G(x, x, y) \preceq G(x, y, z)$, whenever $y \neq z$, for all $x, y, z \in X$,

(GBC4) $G(x, y, z)=G(y, x, z)=\cdots \quad$ (symmetric in all three variables),

$(G B C 5) G(x, y, z) \preceq s(G(x, a, a)+G(a, y, z))$, for all $x, y, z, a \in X$ (the rectangle inequality).

Then the pair $(X, G)$ is called a generalized b-cone metric space or, more specifically, a $G_{b}$-cone metric space.

The concept of a $G_{b}$-cone metric space is more general than that of a $G_{b}$-metric space, a $G$-cone metric space, and a cone metric space. For the definition of $G_{b}$-metric, $G$-cone metric, cone metric spaces, and related concepts we refer the reader to [15], [10], [18]. 
Definition 3. [3] Let $(X, G)$ be a $G_{b}$-cone metric space. A sequence $\left(x_{n}\right)$ in $X$ is said to be

(1) a $G_{b}$-cone Cauchy sequence if, for every $c \in E$ with $\theta \ll c$, there exists $n_{0} \in \mathbb{N}$ such that for all $n, m, l>n_{0}, G\left(x_{n}, x_{m}, x_{l}\right) \ll c$.

(2) a $G_{b}$-cone convergent sequence if, for every $c \in E$ with $\theta \ll c$, there exists $n_{0} \in \mathbb{N}$ such that for all $m, n>n_{0}, G\left(x_{n}, x_{m}, x\right) \ll \theta$ for some fixed $x$ in $X$. Here $x$ is called the $G_{b}$-limit of $\left(x_{n}\right)$ and is denoted by $G_{b^{-}} \lim _{n \rightarrow+\infty} x_{n}=x$ or $x_{n} \rightarrow x$ as $n \rightarrow+\infty$.

Definition 4. [3] $A G_{b}$-cone metric space $X$ is a $G_{b}$-complete cone metric space, if every $G_{b}$-cone Cauchy sequence in $X$ is $G_{b}$-cone convergent in $X$.

Next, we state some $G_{b}$-cone convergence results.

Proposition 1. [3] Let $(X, G)$ be a $G_{b}$-cone metric space. Then the following conditions are equivalent

(1) $\left(x_{n}\right)$ is $G_{b}$-cone Cauchy in $X$.

(2) For every $c \in E$ with $\theta \ll c$, there is $n_{0} \in \mathbb{N}$ such that for all $n, m>n_{0}, G\left(x_{n}, x_{m}, x_{m}\right) \ll$ $\theta$.

Lemma 2. [3] Let $(X, G)$ be a $G_{b}$-cone metric space and $P$ a normal cone with normal constant $k$. A sequence $\left(x_{n}\right) \subset X$ is $G_{b}$-cone convergent to $x$ if and only if $G\left(x_{n}, x_{m}, x\right) \rightarrow$ $\theta$, as $n, m \rightarrow+\infty$.

Proposition 2. [3] Let $(X, G)$ be a $G_{b}$-cone metric space and $P$ be a normal cone with normal constant $k$. The following conditions are equivalent

(1) $\left(x_{n}\right)$ is $G_{b}$-cone convergent to $x$,

(2) $G\left(x_{n}, x_{n}, x\right) \rightarrow \theta$, as $n \rightarrow+\infty$,

(3) $G\left(x_{n}, x, x\right) \rightarrow \theta$, as $n \rightarrow+\infty$,

(4) $G\left(x_{m}, x_{n}, x\right) \rightarrow \theta$, as $n, m \rightarrow+\infty$.

Lemma 3. [3] Let $(X, G)$ be a complete $G_{b}$-cone metric space and $P$ be a normal cone with normal constant $k$. If $\left(x_{n}\right) \subset X G_{b}$-cone converges to $x$ and $G_{b}$-cone converges to $y$, then $x=y$ (the limit of $\left(x_{n}\right)$ is unique).

Proposition 3. [3] Let $(X, G)$ be a $G_{b}$-cone metric space and $P$ be a normal cone with normal constant $k$. Then sequence $\left(x_{n}\right)$ is $G_{b}$-cone Cauchy if and only if $G\left(x_{n}, x_{m}, x_{l}\right) \rightarrow$ $\theta$, as $n, m, l \rightarrow+\infty$.

Definition 5. Let $f$ and $F$ be self mappings of a set $X$. If $y=f x=F x$ for some $x$ in $X$, then $x$ is called a coincidence point of $f$ and $F$ and $y$ is called a point of coincidence of $f$ and $F$.

Definition 6. The self-mappings $f$ and $F$ of a set $X$ are said to be weakly compatible if they commute at their coincidence points, that is, if $f u=F u$ for some $u$ in $X$, then $f F u=F f u$.

Proposition 4. [7] Let $f$ and $F$ be weakly compatible self mappings of a set $X$. If $f$ and $F$ have a unique point of coincidence, that is, $y=f x=F x$, then $y$ is the unique common fixed point of $f$ and $F$. 


\section{Main results}

Our existence first results for coincidence common fixed points no condition of normality assumed.

Theorem 1. Let $(X, G)$ be a cone $G_{b}$-metric space with the coefficient $s \geq 1$ relative to a solid cone $P$. Suppose that the mappings $F, T, R, f: X \rightarrow X$ satisfy the condition that for some constant $\lambda \in\left[0, \frac{1}{2}\right)$ and for all $x, y, z \in X$, there exists

$$
\begin{aligned}
M(x, y, z) \in & \{G(f x, f y, f z), G(F x, f y, f z), G(f x, T y, f z), G(f x, f y, R z), \\
& G(f x, T y, R z), G(F x, f y, R z), G(F x, T y, f z), G(F x, F x, f x), \\
& G(T y, T y, f y), G(R z, R z, f z)\}
\end{aligned}
$$

such that

$$
s^{2} G(F x, T y, R z) \preceq \lambda M(x, y, z) .
$$

If $F(X) \cup T(X) \cup R(X) \subset f(X)$ and $f(X)$ is a $G_{b}$-complete subspace of $X$, then $F, T, R$ and $f$ have a unique point of coincidence in $X$. If the pairs $(f, F),(f, T)$ and $(f, R)$ are further weakly compatible, then $F, T, R$ and $f$ have a unique common fixed point.

Proof. Let $x_{0}$ in $X$ be an arbitrary point. Since $F(X) \cup T(X) \cup R(X) \subset f(X)$, there exist two sequences $\left(x_{n}\right)$ and $\left(y_{n}\right)$ in $X$ such that

$$
y_{3 n}=f x_{3 n+1}=F x_{3 n}, \quad y_{3 n+1}=f x_{3 n+2}=T x_{3 n+1}, \quad y_{3 n+2}=f x_{3 n+3}=R x_{3 n+2},
$$

for all $n \in \mathbb{N}$. By the contractive condition, for all $n \in \mathbb{N}$, there exists

$$
\begin{aligned}
M\left(x_{3 n}, x_{3 n+1}, x_{3 n+2}\right) \in & \left\{G\left(f x_{3 n}, f x_{3 n+1}, f x_{3 n+2}\right), G\left(F x_{3 n}, f x_{3 n+1}, f x_{3 n+2}\right),\right. \\
& G\left(f x_{3 n}, T x_{3 n+1}, f x_{3 n+2}\right), G\left(f x_{3 n}, f x_{3 n+1}, R x_{3 n+2}\right), \\
& G\left(f x_{3 n}, T x_{3 n+1}, R x_{3 n+2}\right), G\left(F x_{3 n}, f x_{3 n+1}, R x_{3 n+2}\right), \\
& G\left(F x_{3 n}, T x_{3 n+1}, f x_{3 n+2}\right), G\left(F x_{3 n}, F x_{3 n}, f x_{3 n}\right), \\
& \left.G\left(T x_{3 n+1}, T x_{3 n+1}, f x_{3 n+1}\right), G\left(R x_{3 n+2}, R x_{3 n+2}, f x_{3 n+2}\right)\right\} \\
= & \left\{G\left(y_{3 n-1}, y_{3 n}, y_{3 n+1}\right), G\left(y_{3 n}, y_{3 n}, y_{3 n+1}\right),\right. \\
& G\left(y_{3 n-1}, y_{3 n+1}, y_{3 n+1}\right), G\left(y_{3 n-1}, y_{3 n}, y_{3 n+2}\right), \\
& G\left(y_{3 n-1}, y_{3 n+1}, y_{3 n+2}\right), G\left(y_{3 n}, y_{3 n}, y_{3 n+2}\right), \\
& G\left(y_{3 n}, y_{3 n+1}, y_{3 n+1}\right), G\left(y_{3 n}, y_{3 n}, y_{3 n-1}\right), \\
& \left.G\left(y_{3 n+1}, y_{3 n+1}, y_{3 n}\right), G\left(y_{3 n+2}, y_{3 n+2}, y_{3 n+1}\right)\right\}
\end{aligned}
$$

such that

$$
\begin{aligned}
G\left(y_{3 n}, y_{3 n+1}, y_{3 n+2}\right) & =G\left(F x_{3 n}, T x_{3 n+1}, R x_{3 n+2}\right) \\
& \preceq \frac{\lambda}{s^{2}} M\left(x_{3 n}, x_{3 n+1}, x_{3 n+2}\right) .
\end{aligned}
$$

We discuss three cases.

Case 1. $M\left(x_{3 n}, x_{3 n+1}, x_{3 n+2}\right) \in\left\{G\left(y_{3 n}, y_{3 n}, y_{3 n+2}\right), G\left(y_{3 n+2}, y_{3 n+2}, y_{3 n+1}\right)\right\}$. By (GBC3) and (GBC4),

$$
G\left(y_{3 n}, y_{3 n+1}, y_{3 n+2}\right) \preceq \lambda G\left(y_{3 n}, y_{3 n+1}, y_{3 n+2}\right) .
$$


By Lemma 1 (PT6) and (GBC1), $y_{3 n}=y_{3 n+1}=y_{3 n+2}$. By applying the contractive condition, there exists

$$
\begin{aligned}
M\left(x_{3 n+3}, x_{3 n+1}, x_{3 n+2}\right) \in & \left\{G\left(f x_{3 n+3}, f x_{3 n+1}, f x_{3 n+2}\right), G\left(F x_{3 n+3}, f x_{3 n+1}, f x_{3 n+2}\right),\right. \\
& G\left(f x_{3 n+3}, T x_{3 n+1}, f x_{3 n+2}\right), G\left(f x_{3 n+3}, f x_{3 n+1}, R x_{3 n+2}\right), \\
& G\left(f x_{3 n+3}, T x_{3 n+1}, R x_{3 n+2}\right), G\left(F x_{3 n+3}, f x_{3 n+1}, R x_{3 n+2}\right), \\
& G\left(F x_{3 n+3}, T x_{3 n+1}, f x_{3 n+2}\right), G\left(F x_{3 n+3}, F x_{3 n+3}, f x_{3 n+3}\right), \\
& \left.G\left(T x_{3 n+1}, T x_{3 n+1}, f x_{3 n+1}\right), G\left(R x_{3 n+2}, R x_{3 n+2}, f x_{3 n+2}\right)\right\} \\
= & \left\{G\left(y_{3 n+2}, y_{3 n}, y_{3 n+1}\right), G\left(y_{3 n+3}, y_{3 n}, y_{3 n+1}\right),\right. \\
& G\left(y_{3 n+2}, y_{3 n+1}, y_{3 n+1}\right), G\left(y_{3 n+2}, y_{3 n}, y_{3 n+2}\right), \\
& G\left(y_{3 n+2}, y_{3 n+1}, y_{3 n+2}\right), G\left(y_{3 n+3}, y_{3 n}, y_{3 n+2}\right), \\
& G\left(y_{3 n+3}, y_{3 n+1}, y_{3 n+1}\right), G\left(y_{3 n+3}, y_{3 n+3}, y_{3 n+2}\right), \\
& \left.G\left(y_{3 n+1}, y_{3 n+1}, y_{3 n}\right), G\left(y_{3 n+2}, y_{3 n+2}, y_{3 n+1}\right)\right\} \\
= & \left\{\theta, G\left(y_{3 n+3}, y_{3 n+2}, y_{3 n+2}\right), \theta, \theta, \theta, G\left(y_{3 n+3}, y_{3 n+2}, y_{3 n+2}\right),\right. \\
& \left.G\left(y_{3 n+3}, y_{3 n+2}, y_{3 n+2}\right), G\left(y_{3 n+3}, y_{3 n+3}, y_{3 n+2}\right), \theta, \theta\right\}
\end{aligned}
$$

such that

$$
\begin{aligned}
G\left(y_{3 n+3}, y_{3 n+1}, y_{3 n+2}\right) & =G\left(F x_{3 n+3}, T x_{3 n+1}, R x_{3 n+2}\right) \\
& \preceq \frac{\lambda}{s^{2}} M\left(x_{3 n+3}, x_{3 n+1}, x_{3 n+2}\right) .
\end{aligned}
$$

Hence

$$
G\left(y_{3 n+3}, y_{3 n+1}, y_{3 n+2}\right) \preceq \frac{\lambda}{s^{2}} G\left(y_{3 n+3}, y_{3 n+1}, y_{3 n+2}\right) .
$$

By a similar argument, we obtain $y_{3 n+2}=y_{3 n+3}$. Thus $\left(y_{n}\right)$ is a constant sequence, which implies that $\left(y_{n}\right)$ is a $G_{b}$-Cauchy sequence.

Case 2. $M\left(x_{3 n}, x_{3 n+1}, x_{3 n+2}\right) \in\left\{G\left(y_{3 n-1}, y_{3 n}, y_{3 n+1}\right), G\left(y_{3 n}, y_{3 n}, y_{3 n+1}\right)\right.$,

$\left.G\left(y_{3 n-1}, y_{3 n+1}, y_{3 n+1}\right), G\left(y_{3 n}, y_{3 n+1}, y_{3 n+1}\right), G\left(y_{3 n}, y_{3 n}, y_{3 n-1}\right), G\left(y_{3 n+1}, y_{3 n+1}, y_{3 n}\right)\right\}$.

By (GBC3) and (GBC4),

$$
G\left(y_{3 n}, y_{3 n+1}, y_{3 n+2}\right) \preceq \frac{\lambda}{s^{2}} G\left(y_{3 n-1}, y_{3 n}, y_{3 n+1}\right) .
$$

Case 3. $M\left(x_{3 n}, x_{3 n+1}, x_{3 n+2}\right) \in\left\{G\left(y_{3 n-1}, y_{3 n}, y_{3 n+2}\right), G\left(y_{3 n-1}, y_{3 n+1}, y_{3 n+2}\right)\right\}$. Assumptions (GBC5), (GBC3) and (GBC4) imply

$$
G\left(y_{3 n}, y_{3 n+1}, y_{3 n+2}\right) \preceq \frac{\lambda}{s-\lambda} G\left(y_{3 n-1}, y_{3 n}, y_{3 n+1}\right) .
$$

Hence for all $n \in \mathbb{N}$,

$$
G\left(y_{3 n}, y_{3 n+1}, y_{3 n+2}\right) \preceq \alpha G\left(y_{3 n-1}, y_{3 n}, y_{3 n+1}\right),
$$

where $\alpha=\max \left\{\frac{\lambda}{s-\lambda}, \frac{\lambda}{s^{2}}\right\}=\frac{\lambda}{s-\lambda} \in[0,1)$. Similarly we can prove that

$$
G\left(y_{3 n+1}, y_{3 n+2}, y_{3 n+3}\right) \preceq \alpha G\left(y_{3 n}, y_{3 n+1}, y_{3 n+2}\right)
$$

and

$$
G\left(y_{3 n+2}, y_{3 n+3}, y_{3 n+4}\right) \preceq \alpha G\left(y_{3 n+1}, y_{3 n+2}, y_{3 n+3}\right) .
$$


From (1), (2), and (3) that, for all $n \in \mathbb{N}$, we get

$$
G\left(y_{n}, y_{n+1}, y_{n+2}\right) \preceq \alpha G\left(y_{n-1}, y_{n}, y_{n+1}\right) .
$$

From the inequality in (4), we infer that for all $n \in \mathbb{N}$,

$$
G\left(y_{n}, y_{n+1}, y_{n+2}\right) \preceq \alpha G\left(y_{n-1}, y_{n}, y_{n+1}\right) \preceq \cdots \preceq \alpha^{n} G\left(y_{0}, y_{1}, y_{2}\right) .
$$

For every $n, m \in \mathbb{N}$ with $m>n$, using (GBC5), (GBC3), (GBC4), and (5), we deduce that

$$
\begin{aligned}
G\left(y_{n}, y_{m}, y_{m}\right) \preceq & s G\left(y_{n}, y_{n+1}, y_{n+1}\right)+s^{2} G\left(y_{n+1}, y_{n+2}, y_{n+2}\right)+\cdots+s^{m-n} \\
& G\left(y_{m-1}, y_{m}, y_{m}\right) \\
\preceq & s G\left(y_{n}, y_{n+1}, y_{n+2}\right)+s^{2} G\left(y_{n+1}, y_{n+2}, y_{n+3}\right)+\cdots+s^{m-n} \\
& G\left(y_{m-1}, y_{m}, y_{m+1}\right) \\
\preceq & s \alpha^{n} G\left(y_{0}, y_{1}, y_{2}\right)+s^{2} \alpha^{n+1} G\left(y_{0}, y_{1}, y_{2}\right)+\cdots+s^{m-n} \alpha^{m-1} \\
& G\left(y_{0}, y_{1}, y_{2}\right) \\
= & s \alpha^{n} G\left(y_{0}, y_{1}, y_{2}\right)\left(1+s \alpha+\cdots+(s \alpha)^{m-n-1}\right) \\
\preceq & \frac{s \alpha^{n}}{1-s \alpha} G\left(y_{0}, y_{1}, y_{2}\right) \rightarrow \theta, \text { as } n \rightarrow+\infty .
\end{aligned}
$$

By Lemma 1 (PT7), for every $c \in E$ with $c \gg \theta$, there exists $n_{0} \in \mathbb{N}$ such that for all $n>n_{0}$,

$$
\frac{s \alpha^{n}}{1-s \alpha} G\left(y_{0}, y_{1}, y_{2}\right) \ll c .
$$

Moreover, for any $m>n>n_{0}$, by Lemma 1 (PT1),

$$
G\left(y_{n}, y_{m}, y_{m}\right) \ll c .
$$

Using Proposition 1 , we conclude that $\left(y_{n}\right)$ is a $G_{b}$-Cauchy sequence in $f(X)$. Since $f(X)$ is $G_{b}$-complete subspace of $X$, there exists $v \in f(X)$ such that $f\left(x_{n}\right) \rightarrow v$, as $n \rightarrow+\infty$. Consequently, there is an $u \in X$ such that $f u=v$. Since the sequences $\left(f x_{3 n+1}\right)=\left(F x_{3 n}\right),\left(f x_{3 n+2}\right)=\left(T x_{3 n+1}\right)$, and $\left(f x_{3 n+3}\right)=\left(R x_{3 n+2}\right)$ are subsequences of $\left(y_{n}\right)$, they converge to $u$. Next we prove that $f u=F u$. By (GBC5) and (GBC3), we have for all $n \in \mathbb{N}$

$$
\begin{aligned}
G(F u, f u, f u) & \preceq s G\left(F u, y_{3 n+1}, y_{3 n+1}\right)+s G\left(y_{3 n+1}, f u, f u\right) \\
& \preceq s G\left(F u, y_{3 n+1}, y_{3 n+2}\right)+s G\left(y_{3 n+1}, f u, f u\right) .
\end{aligned}
$$

The contractive condition yields that for all $n \in \mathbb{N}$, there exists

$$
\begin{aligned}
M\left(u, x_{3 n+1}, x_{3 n+2}\right) \in & \left\{G\left(f u, f x_{3 n+1}, f x_{3 n+2}\right), G\left(F u, f x_{3 n+1}, f x_{3 n+2}\right),\right. \\
& G\left(f u, T x_{3 n+1}, f x_{3 n+2}\right), G\left(f u, f x_{3 n+1}, R x_{3 n+2}\right), \\
& G\left(f u, T x_{3 n+1}, R x_{3 n+2}\right), G\left(F u, f x_{3 n+1}, R x_{3 n+2}\right), \\
& G\left(F u, T x_{3 n+1}, f x_{3 n+2}\right), G(F u, F u, f u), \\
& \left.G\left(T x_{3 n+1}, T x_{3 n+1}, f x_{3 n+1}\right), G\left(R x_{3 n+2}, R x_{3 n+2}, f x_{3 n+2}\right)\right\} \\
= & \left\{G\left(f u, y_{3 n}, y_{3 n+1}\right), G\left(F u, y_{3 n}, y_{3 n+1}\right),\right. \\
& G\left(f u, y_{3 n+1}, y_{3 n+1}\right), G\left(f u, y_{3 n}, y_{3 n+2}\right), \\
& G\left(f u, y_{3 n+1}, y_{3 n+2}\right), G\left(F u, y_{3 n}, y_{3 n+2}\right), \\
& G\left(F u, y_{3 n+1}, y_{3 n+1}\right) G(F u, F u, f u), \\
& \left.G\left(y_{3 n+1}, y_{3 n+1}, y_{3 n}\right), G\left(y_{3 n+2}, y_{3 n+2}, y_{3 n+1}\right)\right\}
\end{aligned}
$$


such that

$$
\begin{aligned}
G(F u, f u, f u) & \preceq s G\left(F u, T x_{3 n+1}, R x_{3 n+2}\right)+s G\left(y_{3 n+1}, f u, f u\right) \\
& \preceq \frac{\lambda}{s} M\left(u, x_{3 n+1}, x_{3 n+2}\right)+s G\left(y_{3 n+1}, f u, f u\right) .
\end{aligned}
$$

We distinguish between three cases.

Case 1. $M\left(u, x_{3 n+1}, x_{3 n+2}\right) \in\left\{G\left(f u, y_{3 n}, y_{3 n+1}\right), G\left(f u, y_{3 n+1}, y_{3 n+1}\right)\right.$,

$\left.G\left(f u, y_{3 n}, y_{3 n+2}\right), G\left(f u, y_{3 n+1}, y_{3 n+2}\right), G\left(y_{3 n+1}, y_{3 n+1}, y_{3 n}\right), G\left(y_{3 n+2}, y_{3 n+2}, y_{3 n+1}\right)\right\}$

If, e.g., $M\left(u, x_{3 n+1}, x_{3 n+2}\right)=G\left(f u, y_{3 n}, y_{3 n+1}\right)$, then for all $n \in \mathbb{N}$,

$$
G(F u, f u, f u) \preceq \frac{\lambda}{s} G\left(f u, y_{3 n}, y_{3 n+1}\right)+s G\left(y_{3 n+1}, f u, f u\right) .
$$

Since $y_{n} \rightarrow f u$, as $n \rightarrow+\infty$, then for $c \gg \theta$, there exists $n_{0} \in \mathbb{N}$ such that for all $n>n_{0}$,

$$
G\left(f u, y_{3 n}, y_{3 n+1}\right) \ll \frac{s c}{2 \lambda}, G\left(y_{3 n+1}, f u, f u\right) \ll \frac{c}{2 s} .
$$

Hence

$$
\theta \preceq G(F u, f u, f u) \preceq \frac{\lambda}{s} G\left(f u, y_{3 n}, y_{3 n+1}\right)+s G\left(y_{3 n+1}, f u, f u\right) \ll c .
$$

By Lemma 1 (PT4), $G(F u, f u, f u)=\theta$, that is $F u=f u$.

The remaining cases are readily dealt with in a similar way.

Case 2. $M\left(u, x_{3 n+1}, x_{3 n+2}\right) \in\left\{G\left(F u, y_{3 n}, y_{3 n+1}\right), G\left(F u, y_{3 n}, y_{3 n+2}\right)\right.$,

$\left.G\left(F u, y_{3 n+1}, y_{3 n+1}\right)\right\}$.

If $M\left(u, x_{3 n+1}, x_{3 n+2}\right)=G\left(F u, y_{3 n}, y_{3 n+1}\right)$, then by $(\mathrm{GBC} 5)$, for all $n \in \mathbb{N}$, we get

$$
\begin{aligned}
G(F u, f u, f u) & \preceq \frac{\lambda}{s} G\left(F u, y_{3 n}, y_{3 n+1}\right)+s G\left(y_{3 n+1}, f u, f u\right) \\
& \preceq \lambda G(F u, f u, f u)+\lambda G\left(f u, y_{3 n}, y_{3 n+1}\right)+s G\left(y_{3 n+1}, f u, f u\right),
\end{aligned}
$$

which implies that

$$
(1-\lambda) G(F u, f u, f u) \preceq \lambda G\left(f u, y_{3 n}, y_{3 n+1}\right)+s G\left(y_{3 n+1}, f u, f u\right) .
$$

Since $y_{n} \rightarrow f u$ as $n \rightarrow+\infty$, then for $c \gg \theta$, there exists $n_{0} \in \mathbb{N}$ such that for all $n>n_{0}$,

$$
G\left(f u, y_{3 n}, y_{3 n+1}\right) \ll \frac{c}{2 \lambda}, G\left(y_{3 n+1}, f u, f u\right) \ll \frac{c}{2 s} .
$$

Hence

$$
\theta \preceq(1-\lambda) G(F u, f u, f u) \preceq \lambda G\left(f u, y_{3 n}, y_{3 n+1}\right)+s G\left(y_{3 n+1}, f u, f u\right) \ll c .
$$

By Lemma 1 (PT4), $(1-\lambda) G(F u, f u, f u)=\theta$, that is $F u=f u$.

The remaining cases are analogous.

Case 3. $M\left(u, x_{3 n+1}, x_{3 n+2}\right)=G(F u, F u, f u)$. By (GBC5) and (GBC4), for all $n \in \mathbb{N}$, we have

$$
(1-2 \lambda) G(F u, f u, f u) \preceq s G\left(y_{3 n+1}, f u, f u\right) .
$$


Since $y_{n} \rightarrow f u$ as $n \rightarrow+\infty$, then for $c \gg \theta$, there exists $n_{0} \in \mathbb{N}$ such that for all $n>n_{0}$,

$$
G\left(y_{3 n+1}, f u, f u\right) \ll \frac{c}{s} .
$$

Hence

$$
\theta \preceq(1-2 \lambda) G(F u, f u, f u) \preceq s G\left(y_{3 n+1}, f u, f u\right) \ll c .
$$

By Lemma 1 (PT4), $(1-2 \lambda) G(F u, f u, f u)=\theta$, that is $F u=f u$.

The other cases are identical. To sum up, $F u=f u$.

We can check that $T u=f u$ and $R u=f u$, too. So,

$$
f u=F u=T u=R u=v,
$$

that is $v$ is a point of coincidence of $f, F, T$ and $R$.

To show that $f, F, T$, and $R$ have a unique point of coincidence in $X$, assume that there exists another coincidence point $v^{\star} \in X$ such that

$$
f u^{\star}=F u^{\star}=T u^{\star}=R u^{\star}=v^{\star},
$$

for some $u^{\star} \in X$. By the contractive condition, there exists

$$
\begin{aligned}
M\left(u^{\star} u, u\right) \in & \left\{G\left(f u^{\star}, f u, f u\right), G\left(F u^{\star}, f u, f u\right), G\left(f u^{\star}, T u, f u\right), G\left(f u^{\star}, f u, R u\right),\right. \\
& G\left(f u^{\star}, T u, R u\right), G\left(F u^{\star}, f u, R u\right), G\left(F u^{\star}, T u, f u\right), G\left(F u^{\star}, F u^{\star}, f u^{\star}\right), \\
& G(T u, T u, f u), G(R u, R u, f u)\} \\
= & \left\{G\left(v^{\star}, v, v\right)\right\}
\end{aligned}
$$

such that

$$
\begin{aligned}
G\left(v^{\star}, v, v\right) & =G\left(F u^{\star}, T u, R u\right) \\
& \preceq \frac{\lambda}{s^{2}} M\left(u^{\star} u, u\right) .
\end{aligned}
$$

Therefore,

$$
G\left(v^{\star}, v, v\right) \preceq \frac{\lambda}{s^{2}} G\left(v^{\star}, v, v\right) .
$$

By Lemma 1 (PT6), $G\left(v^{\star}, v, v\right)=\theta$, that is $v^{\star}=v$. Finally, since the pairs $(f, F),(f, T)$ and $(f, R)$ are weakly compatible, we have

$$
F f u=f F u, T f u=f T u, R f u=f R u .
$$

This implies that $F v=T v=R v=f v=t$, i.e., $t$ is a point of coincidence of $f, F, T$ and $R$.So $t=v$ by uniqueness. Finally Proposition 4 tells us that $v$ is the unique common fixed point of $f, F, T$, and $R$.

Theorem 1 is illustrated by the following example inspired from [1, Example 2.12].

Example 1. Let $X=[0,1], E=C[0,1]$ be endowed with the strongly locally convex topology $\tau\left(E, E^{*}\right)$ and let $P=\{x \in E: x(t) \geq 0, t \in[0,1]\}$. Then the cone is $\tau\left(E, E^{*}\right)$ solid but not normal with respect to the topology $\tau\left(E, E^{*}\right)$. Define the mapping $G: X \times$ $X \times X \rightarrow E$ by

$$
G(x, y, z)(t)=\max \left\{|x-y|^{2},|y-z|^{2},|x-z|^{2}\right\} e^{t} .
$$


Then $G$ is a $G_{b}$-cone metric on $X$ with the coefficient $s=2$. On $X$, define the self-maps $f, F, T$, and $R$ by

$$
\begin{aligned}
& F(x)= \begin{cases}\frac{1}{4}, & x \in\left[0, \frac{2}{3}\right), \\
\frac{1}{7}, & x \in\left[\frac{2}{3}, 1\right],\end{cases} \\
& T(x)= \begin{cases}\frac{1}{4}, & x \in\left[0, \frac{2}{3}\right), \\
\frac{1}{5}, & x \in\left[\frac{2}{3}, 1\right],\end{cases} \\
& R(x)= \begin{cases}\frac{1}{4}, & x \in\left[0, \frac{2}{3}\right), \\
\frac{1}{6}, & x \in\left[\frac{2}{3}, 1\right],\end{cases} \\
& f(x)= \begin{cases}x, & x \in\left[0, \frac{2}{3}\right), \\
\frac{2}{3}, & x \in\left[\frac{2}{3}, 1\right] .\end{cases}
\end{aligned}
$$

Note that for all $x, y, z \in X$, there exists

$$
\begin{aligned}
M(x, y, z) \in & \{G(f x, f y, f z), G(F x, f y, f z), G(f x, T y, f z), G(f x, f y, R z), \\
& G(f x, T y, R z), G(F x, f y, R z), G(F x, T y, f z), G(F x, F x, f x), \\
& G(T y, T y, f y), G(R z, R z, f z)\}
\end{aligned}
$$

such that

$$
4 G(F x, T y, R z) e^{t} \leq \frac{2}{5} M(x, y, z) e^{t} .
$$

Moreover $f(X)=\left[0, \frac{2}{3}\right]$ is a $G_{b}$-complete subspace of $X, F(X) \cup T(X) \cup R(X) \subset f(X)$, and the pairs $(f, F),(f, T)$, and $(f, R)$ are weakly compatible. Therefore all the conditions of Theorem 1 are fulfilled. Finally, $\frac{1}{4}$ is the unique point of coincidence and the unique common fixed point for all of the mappings $F, T, R$, and $f$.

The following result is immediately derived from Theorem 1.

Corollary 1. Let $(X, G)$ be a cone $G$-metric space relative to a solid cone P. Suppose that the mappings $F, T, R, f: X \rightarrow X$ satisfy for some constant $\lambda \in\left[0, \frac{1}{2}\right)$ and for all $x, y, z \in X$, there exists

$$
\begin{aligned}
M(x, y, z) \in & \{G(f x, f y, f z), G(F x, f y, f z), G(f x, T y, f z), G(f x, f y, R z), \\
& G(f x, T y, R z), G(F x, f y, R z), G(F x, T y, f z), G(F x, F x, f x), \\
& G(T y, T y, f y), G(R z, R z, f z)\}
\end{aligned}
$$

such that

$$
G(F x, T y, R z) \preceq \lambda M(x, y, z) .
$$

If $F(X) \cup T(X) \cup R(X) \subset f(X)$ and $f(X)$ is a $G$-complete subspace of $X$, then $F, T, R$ and $f$ have a unique point of coincidence in $X$. If further the pairs $(f, F),(f, T)$, and $(f, R)$ are weakly compatible, then $F, T, R$ and $f$ have a unique common fixed point. 
For $x, y, z \in X$, the distance $G_{k}(x, y, z)$ between $x, y$ and $z$ is defined by

$$
G_{k}(x, y, z)=\|G(x, y, z)\| .
$$

When the assumption of normality is assumed, the existence results for coincidence common fixed points are collected in the following

Theorem 2. Let $(X, G)$ be a cone $G_{b}$-metric space with the coefficient $s \geq 1$ relative to a normal constant $k \geq 1$. Suppose that the mappings $F, T, R, f: X \rightarrow X$ satisfy

$$
s G_{k}(F x, T y, R z) \leq \lambda M_{k}(x, y, z)+L N_{k}(x, y, z),
$$

where

$$
\begin{aligned}
M_{k}(x, y, z)= & \max \left\{G_{k}(f x, f y, f z), G_{k}(F x, f y, f z), G_{k}(f x, T y, f z), G_{k}(f x, f y, R z),\right. \\
& G_{k}(f x, T y, R z), G_{k}(F x, f y, R z), G_{k}(F x, T y, f z), G_{k}(F x, F x, f x), \\
& G_{k}(T y, T y, f y), G_{k}(R z, R z, f z), G_{k}(F x, f x, f x), G_{k}(T y, f y, f y), \\
& \left.G_{k}(R z, f z, f z)\right\}, \\
N_{k}(x, y, z)= & \min \left\{G_{k}(F x, f y, f y), G_{k}(F x, f z, f z), G_{k}(T y, f z, f z), G_{k}(T y, f x, f x),\right. \\
& \left.G_{k}(R z, f x, f x), G_{k}(R z, f y, f y)\right\},
\end{aligned}
$$

for all $x, y, z \in X, \lambda \in\left[0, \frac{1}{2 k}\right)$, and $L \geq 0$. If $F(X) \cup T(X) \cup R(X) \subset f(X)$ and $f(X)$ is a $G_{b}$-complete subspace of $X$, then $F, T, R$, and $f$ have a unique point of coincidence in $X$. Moreover if the pairs $(f, F),(f, T)$, and $(f, R)$ are weakly compatible, then $F, T, R$ and $f$ have a unique common fixed point.

Proof. Let $x_{0}$ in $X$ be an arbitrary point since $F(X) \cup T(X) \cup R(X) \subset f(X)$. There exist sequences $\left(x_{n}\right)$ and $\left(y_{n}\right)$ in $X$ such that

$$
y_{3 n}=f x_{3 n+1}=F x_{3 n}, \quad y_{3 n+1}=f x_{3 n+2}=T x_{3 n+1}, \quad y_{3 n+2}=f x_{3 n+3}=R x_{3 n+2},
$$

for all $n=0,1,2, \ldots$. Then from (6), we have for all $n \in \mathbb{N}$

$$
\begin{aligned}
G_{k}\left(y_{3 n}, y_{3 n+1}, y_{3 n+2}\right) & =G_{k}\left(F x_{3 n}, T x_{3 n+1}, R x_{3 n+2}\right) \\
& \leq \frac{\lambda}{s} M_{k}\left(x_{3 n}, x_{3 n+1}, x_{3 n+2}\right)+\frac{L}{s^{2}} N_{k}\left(x_{3 n}, x_{3 n+1}, x_{3 n+2}\right),
\end{aligned}
$$


where

$$
\begin{aligned}
M_{k}\left(x_{3 n}, x_{3 n+1}, x_{3 n+2}\right)= & \max \left\{G_{k}\left(f x_{3 n}, f x_{3 n+1}, f x_{3 n+2}\right), G_{k}\left(F x_{3 n}, f x_{3 n+1}, f x_{3 n+2}\right),\right. \\
& G_{k}\left(f x_{3 n}, T x_{3 n+1}, f x_{3 n+2}\right), G_{k}\left(f x_{3 n}, f x_{3 n+1}, R x_{3 n+2}\right), \\
& G_{k}\left(f x_{3 n}, T x_{3 n+1}, R x_{3 n+2}\right), G_{k}\left(F x_{3 n}, f x_{3 n+1}, R x_{3 n+2}\right), \\
& G_{k}\left(F x_{3 n}, T x_{3 n+1}, f x_{3 n+2}\right), G_{k}\left(F x_{3 n}, F x_{3 n}, f x_{3 n}\right), \\
& G_{k}\left(T x_{3 n+1}, T x_{3 n+1}, f x_{3 n+1}\right), G_{k}\left(R x_{3 n+2}, R x_{3 n+2}, f x_{3 n+2}\right) \\
& G_{k}\left(F x_{3 n}, f x_{3 n}, f x_{3 n}\right), G_{k}\left(T x_{3 n+1}, f x_{3 n+1}, f x_{3 n+1}\right), \\
& \left.G_{k}\left(R x_{3 n+2}, f x_{3 n+2}, f x_{3 n+2}\right)\right\} \\
= & \max \left\{G_{k}\left(y_{3 n-1}, y_{3 n}, y_{3 n+1}\right), G_{k}\left(y_{3 n}, y_{3 n}, y_{3 n+1}\right),\right. \\
& G_{k}\left(y_{3 n-1}, y_{3 n+1}, y_{3 n+1}\right), G_{k}\left(y_{3 n-1}, y_{3 n}, y_{3 n+2}\right), \\
& G_{k}\left(y_{3 n-1}, y_{3 n+1}, y_{3 n+2}\right), G_{k}\left(y_{3 n}, y_{3 n}, y_{3 n+2}\right), \\
& G_{k}\left(y_{3 n}, y_{3 n+1}, y_{3 n+1}\right), G_{k}\left(y_{3 n}, y_{3 n}, y_{3 n-1}\right), \\
& G_{k}\left(y_{3 n+1}, y_{3 n+1}, y_{3 n}\right), G_{k}\left(y_{3 n+2}, y_{3 n+2}, y_{3 n+1}\right) \\
& G_{k}\left(y_{3 n}, y_{3 n-1}, y_{3 n-1}\right), G_{k}\left(y_{3 n+1}, y_{3 n}, y_{3 n}\right), \\
& \left.G_{k}\left(y_{3 n+2}, y_{3 n+1}, y_{3 n+1}\right)\right\}
\end{aligned}
$$

and

$$
\begin{aligned}
N_{k}\left(x_{3 n}, x_{3 n+1}, x_{3 n+2}\right)= & \min \left\{G_{k}\left(F x_{3 n}, f x_{3 n+1}, f x_{3 n+1}\right), G_{k}\left(F x_{3 n}, f x_{3 n+2}, f x_{3 n+2}\right),\right. \\
& G_{k}\left(T x_{3 n+1}, f x_{3 n+2}, f x_{3 n+2}\right), G_{k}\left(T x_{3 n+1}, f x_{3 n}, f x_{3 n}\right), \\
& \left.G_{k}\left(R x_{3 n+2}, f x_{3 n}, f x_{3 n}\right), G_{k}\left(R x_{3 n+2}, f x_{3 n+1}, f x_{3 n+1}\right)\right\} \\
= & \min \left\{G_{k}\left(y_{3 n}, y_{3 n}, y_{3 n}\right), G_{k}\left(y_{3 n}, y_{3 n+1}, y_{3 n+1}\right),\right. \\
& G_{k}\left(y_{3 n+1}, y_{3 n+1}, y_{3 n+1}\right), G_{k}\left(y_{3 n+1}, y_{3 n-1}, y_{3 n-1}\right), \\
& \left.G_{k}\left(y_{3 n+2}, y_{3 n-1}, y_{3 n-1}\right), G_{k}\left(y_{3 n+2}, y_{3 n}, y_{3 n}\right)\right\} \\
= & 0 .
\end{aligned}
$$

By (GBC5), (GBC3), and (GBC4), we have

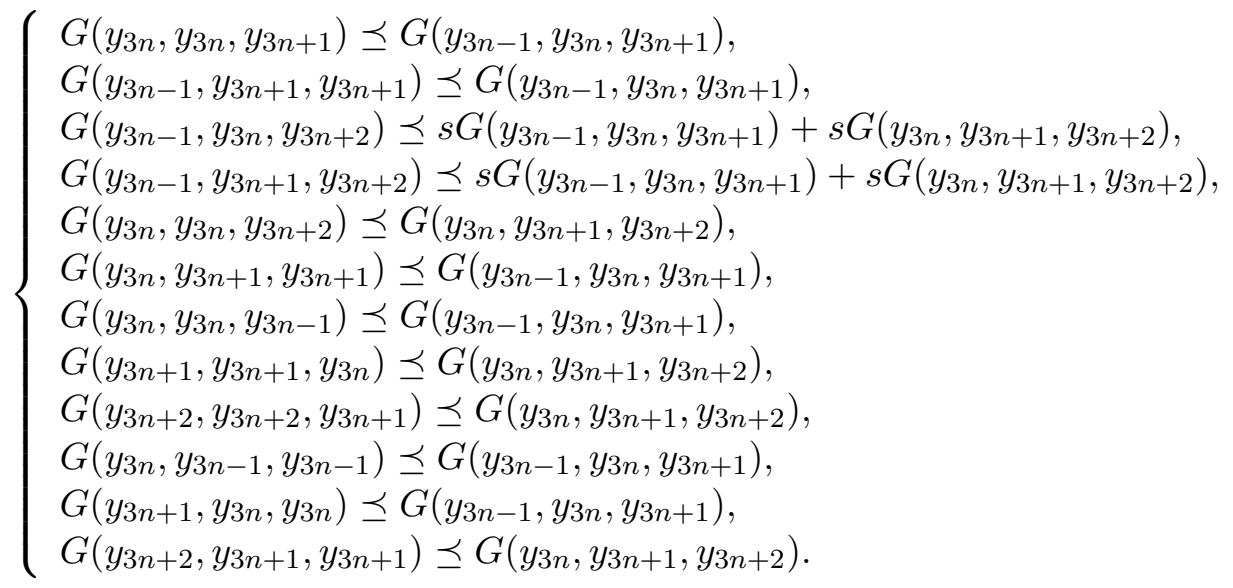

Using the normality of the cone and the fact that $\|\cdot\|$ satisfies the triangle inequality, we 
obtain

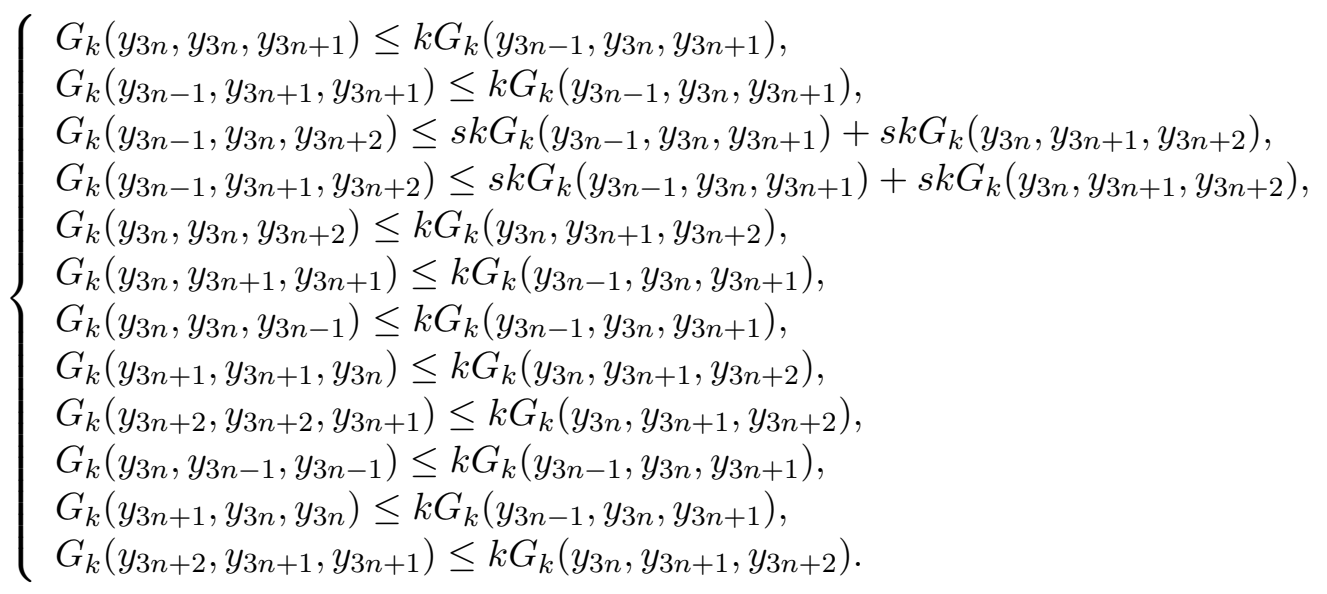

By (7) and (8), for all $n \in \mathbb{N}$

$$
G_{k}\left(y_{3 n}, y_{3 n+1}, y_{3 n+2}\right) \leq \alpha G_{k}\left(y_{3 n-1}, y_{3 n}, y_{3 n+1}\right),
$$

where $\alpha=\frac{k \lambda}{1-k \lambda} \in[0,1)$.

We can also prove that

$$
G_{k}\left(y_{3 n+1}, y_{3 n+2}, y_{3 n+3}\right) \leq \alpha G_{k}\left(y_{3 n}, y_{3 n+1}, y_{3 n+2}\right)
$$

and

$$
G_{k}\left(y_{3 n+2}, y_{3 n+3}, y_{3 n+4}\right) \leq \alpha G_{k}\left(y_{3 n+1}, y_{3 n+2}, y_{3 n+3}\right) .
$$

(9), (10), and (11) imply that, for all $n \in \mathbb{N}$,

$$
G_{k}\left(y_{n}, y_{n+1}, y_{n+2}\right) \leq \alpha G_{k}\left(y_{n-1}, y_{n}, y_{n+1}\right) .
$$

From the inequality in (12), we infer that for all $n \in \mathbb{N}$,

$$
G_{k}\left(y_{n}, y_{n+1}, y_{n+2}\right) \leq \alpha G_{k}\left(y_{n-1}, y_{n}, y_{n+1}\right) \leq \cdots \leq \alpha^{n} G_{k}\left(y_{0}, y_{1}, y_{2}\right) .
$$

Hence for each $n, m, l \in \mathbb{N}$ with $l>m>n$, by (GBC5), (GBC3), and (GBC4), we have

$$
\begin{aligned}
G\left(y_{n}, y_{m}, y_{l}\right) \preceq & s G\left(y_{n}, y_{n+1}, y_{n+1}\right)+s^{2} G\left(y_{n+1}, y_{n+2}, y_{n+2}\right)+\cdots+s^{l-n} \\
\preceq & G\left(y_{l-1}, y_{l}, y_{l}\right) \\
& s\left(y_{n}, y_{n+1}, y_{n+2}\right)+s^{2} G\left(y_{n+1}, y_{n+2}, y_{n+3}\right)+\cdots+s^{l-n} \\
& G\left(y_{l-1}, y_{l}, y_{l+1}\right) .
\end{aligned}
$$

By the normality of the cone, Equation (13), and since $\|$.$\| satisfies the triangle inequality,$ we find

$$
\begin{aligned}
G_{k}\left(y_{n}, y_{m}, y_{l}\right) \leq & k\left(s G_{k}\left(y_{n}, y_{n+1}, y_{n+2}\right)+s^{2} G_{k}\left(y_{n+1}, y_{n+2}, y_{n+3}\right)+\cdots+s^{l-n}\right. \\
& G_{k}\left(y_{l-1}, y_{l}, y_{l+1}\right) \\
\leq & k\left(s \alpha^{n} G_{k}\left(y_{0}, y_{1}, y_{2}\right)+s^{2} \alpha^{n+1} G_{k}\left(y_{0}, y_{1}, y_{2}\right)+\cdots+s^{l-n} \alpha^{l-1}\right. \\
& G_{k}\left(y_{0}, y_{1}, y_{2}\right) \\
= & k s \alpha^{n} G_{k}\left(y_{0}, y_{1}, y_{2}\right)\left(1+s \alpha+\cdots+(s \alpha)^{l-n-1}\right) \\
\leq & \frac{k s \alpha^{n}}{1-s \alpha} G_{k}\left(y_{0}, y_{1}, y_{2}\right) \rightarrow 0, \text { as } n \rightarrow+\infty,
\end{aligned}
$$


which implies that $G\left(y_{n}, y_{m}, y_{l}\right) \rightarrow \theta$, as $n, m, l \rightarrow+\infty$. Proposition 3 implies that $\left(y_{n}\right)$ is a $G_{b}$-Cauchy sequence in $f(X)$. Since $f(X)$ is $G_{b}$-complete subspace of $X$, there exists a point $v$ in $f(X)$ such that $f\left(x_{n}\right) \rightarrow v$ as $n \rightarrow+\infty$. Consequently, there is some $u \in X$ such that $f u=v$. Since the sequences $\left(f x_{3 n+1}\right)=\left(F x_{3 n}\right),\left(f x_{3 n+2}\right)=\left(T x_{3 n+1}\right)$, and $\left(f x_{3 n+3}\right)=\left(R x_{3 n+2}\right)$ are subsequences of $\left(y_{n}\right)$, they converge to the same limit $v$. We show that $f u=F u$. By normality of the cone, Equation (6), and since $\|$.$\| satisfies the$ triangle inequality, for all $n \in \mathbb{N}$, we have

$$
\begin{aligned}
G_{k}\left(F u, y_{3 n+1}, y_{3 n+2}\right) & =G_{k}\left(F u, T x_{3 n+1}, R x_{3 n+2}\right) \\
& \leq \frac{\lambda}{s} M_{k}\left(u, x_{3 n+1}, x_{3 n+2}\right)+\frac{L}{s} N_{k}\left(u, x_{3 n+1}, x_{3 n+2}\right),
\end{aligned}
$$

where

$$
\begin{aligned}
M_{k}\left(u, x_{3 n+1}, x_{3 n+2}\right)= & \max \left\{G_{k}\left(f u, f x_{3 n+1}, f x_{3 n+2}\right), G_{k}\left(F u, f x_{3 n+1}, f x_{3 n+2}\right),\right. \\
& G_{k}\left(f u, T x_{3 n+1}, f x_{3 n+2}\right), G_{k}\left(f u, f x_{3 n+1}, R x_{3 n+2}\right), \\
& G_{k}\left(f u, T x_{3 n+1}, R x_{3 n+2}\right), G_{k}\left(F u, f x_{3 n+1}, R x_{3 n+2}\right), \\
& G_{k}\left(F u, T x_{3 n+1}, f x_{3 n+2}\right), G_{k}(F u, F u, f u), \\
& G_{k}\left(T x_{3 n+1}, T x_{3 n+1}, f x_{3 n+1}\right), G_{k}\left(R x_{3 n+2}, R x_{3 n+2}, f x_{3 n+2}\right), \\
& G_{k}(F u, f u, f u), G_{k}\left(T x_{3 n+1}, f x_{3 n+1}, f x_{3 n+1}\right), \\
& \left.G_{k}\left(R x_{3 n+2}, f x_{3 n+2}, f x_{3 n+2}\right)\right\} \\
= & \max \left\{G_{k}\left(f u, y_{3 n}, y_{3 n+1}\right), G_{k}\left(F u, y_{3 n}, y_{3 n+1}\right),\right. \\
& G_{k}\left(f u, y_{3 n+1}, y_{3 n+1}\right), G_{k}\left(f u, y_{3 n}, y_{3 n+2}\right), \\
& G_{k}\left(f u, y_{3 n+1}, y_{3 n+2}\right), G_{k}\left(F u, y_{3 n}, y_{3 n+2}\right), \\
& G_{k}\left(F u, y_{3 n+1}, y_{3 n+1}\right), G_{k}(F u, F u, f u), \\
& G_{k}\left(y_{3 n+1}, y_{3 n+1}, y_{3 n}\right), G_{k}\left(y_{3 n+2}, y_{3 n+2}, y_{3 n+1}\right), \\
& \left.G_{k}(F u, f u, f u), G_{k}\left(y_{3 n+1}, y_{3 n}, y_{3 n}\right), G_{k}\left(y_{3 n+2}, y_{3 n+1}, y_{3 n+1}\right)\right\}
\end{aligned}
$$

and

$$
\begin{aligned}
N_{k}\left(u, x_{3 n+1}, x_{3 n+2}\right)= & \min \left\{G_{k}\left(F u, f x_{3 n+1}, f x_{3 n+1}\right), G_{k}\left(F u, f x_{3 n+2}, f x_{3 n+2}\right),\right. \\
& G_{k}\left(T x_{3 n+1}, f x_{3 n+2}, f x_{3 n+2}\right), G_{k}\left(T x_{3 n+1}, f u, f u\right), \\
& \left.G_{k}\left(R x_{3 n+2}, f u, f u\right), G_{k}\left(R x_{3 n+2}, f x_{3 n+1}, f x_{3 n+1}\right)\right\} \\
= & \min \left\{G_{k}\left(F u, y_{3 n}, y_{3 n}\right), G_{k}\left(F u, y_{3 n+1}, y_{3 n+1}\right),\right. \\
& G_{k}\left(y_{3 n+1}, y_{3 n+1}, y_{3 n+1}\right), G_{k}\left(y_{3 n+1}, f u, f u\right), \\
& \left.G_{k}\left(y_{3 n+2}, f u, f u\right), G_{k}\left(y_{3 n+2}, y_{3 n}, y_{3 n}\right)\right\} \\
= & 0 .
\end{aligned}
$$

As a consequence,

$$
\begin{aligned}
\lim _{n \rightarrow+\infty} M_{k}\left(u, x_{3 n+1}, x_{3 n+2}\right)= & \max \left\{0, s G_{k}(F u, f u, f u), 0,0,0, s G_{k}(F u, f u, f u),\right. \\
& \left.s G_{k}(F u, f u, f u), G_{k}(F u, F u, f u), 0,0, G_{k}(F u, f u, f u), 0,0\right\} \\
= & \max \left\{s G_{k}(F u, f u, f u), G_{k}(F u, F u, f u)\right\} \\
\leq & \max \left\{s G_{k}(F u, f u, f u), 2 s k G_{k}(F u, f u, f u)\right\} \\
= & 2 s k G_{k}(F u, f u, f u) .
\end{aligned}
$$

As $n \rightarrow+\infty$ in (14), we find

$$
s G_{k}(F u, f u, f u) \leq 2 k \lambda G_{k}(F u, f u, f u) .
$$


Then $G_{k}(F u, f u, f u)=0$ which implies that $G(F u, f u, f u)=\theta$, that is $F u=f u$. In the same way, we can prove that $T u=f u$ and $R u=f u$. Then,

$$
f u=F u=T u=R u=v,
$$

and so $v$ is a point of coincidence of $f, F, T$ and $R$. To show that $f, F, T$ and $R$ have a unique point of coincidence in $X$, assume that there exists a second coincidence point $v^{\star} \in X$ such that

$$
f u^{\star}=F u^{\star}=T u^{\star}=R u^{\star}=v^{\star},
$$

for some $u^{\star} \in X$. Condition (6) yields

$$
\begin{aligned}
G_{k}\left(v^{\star}, v, v\right) & =G_{k}\left(F u^{\star}, T u, R u\right) \\
& \leq \frac{\lambda}{s} M_{k}\left(u^{\star} u, u\right)+\frac{L}{s} N_{k}\left(u^{\star} u, u\right),
\end{aligned}
$$

where

$$
\begin{aligned}
M_{k}\left(u^{\star}, u, u\right)= & \max \left\{G_{k}\left(f u^{\star}, f u, f u\right), G_{k}\left(F u^{\star}, f u, f u\right), G_{k}\left(f u^{\star}, T u, f u\right),\right. \\
& G_{k}\left(f u^{\star}, f u, R u\right), G_{k}\left(f u^{\star}, T u, R u\right), G_{k}\left(F u^{\star}, f u, R u\right), \\
& G_{k}\left(F u^{\star}, T u, f u\right), G_{k}\left(F u^{\star}, F u^{\star}, f u^{\star}\right), G_{k}(T u, T u, f u), \\
& G_{k}(R u, R u, f u), G_{k}\left(F u^{\star}, f u^{\star}, f u^{\star}\right), G_{k}(T u, f u, f u), \\
& \left.G_{k}(R u, f u, f u)\right\} \\
= & G_{k}\left(v^{\star}, v, v\right)
\end{aligned}
$$

and

$$
\begin{aligned}
N_{k}\left(u^{\star}, u, u\right)= & \min \left\{G_{k}\left(F u^{\star}, f u, f u\right), G_{k}\left(F u^{\star}, f u, f u\right), G_{k}(T u, f u, f u),\right. \\
& \left.G_{k}\left(T u, f u^{\star}, f u^{\star}\right), G_{k}\left(R u, f u^{\star}, f u^{\star}\right), G_{k}(R u, f u, f u)\right\} \\
= & 0 .
\end{aligned}
$$

Therefore,

$$
G_{k}\left(v^{\star}, v, v\right) \leq \frac{\lambda}{s} G_{k}\left(v^{\star}, v, v\right) .
$$

So $G_{k}\left(v^{\star}, v, v\right)=0$, which implies that $G\left(v^{\star}, v, v\right)=\theta$, that is $v^{\star}=v$. Finally, since the pairs $(f, F),(f, T)$, and $(f, R)$ are weakly compatible, we obtain

$$
F f u=f F u, T f u=f T u, R f u=f R u .
$$

This implies that $F v=T v=R v=f v=t$, i.e., $t$ is a point of coincidence of $f, F, T$, and $R$ and $t=v$ by uniqueness. Making use of Proposition 4, we conclude that $v$ is the unique common fixed point of $f, F, T$, and $R$.

Example 2. Let $X=[-1,1], E=\mathbb{R}^{2}$ and $P=\left\{(x, y) \in \mathbb{R}^{2}: x, y \geq 0\right\}$. Define $G: X \times X \times X \rightarrow E$ by

$$
G(x, y, z)=\left(\frac{1}{3} \max \left\{|x-y|^{2},|y-z|^{2},|x-z|^{2}\right\}, \frac{2}{3} \max \left\{|x-y|^{2},|y-z|^{2},|x-z|^{2}\right\}\right) .
$$


Then $G$ is a $G_{b}$-cone metric on $X$ with the coefficient $s=2$. Let

$$
G_{k}(x, y, z)=\|G(x, y, z)\|_{1}=\max \left\{|x-y|^{2},|y-z|^{2},|x-z|^{2}\right\} .
$$

Define the self-maps $f, F, T$, and $R$ on $X$ by

$$
\begin{gathered}
F(x)= \begin{cases}-\frac{1}{4}, & x \in\left[-1, \frac{2}{3}\right), \\
-\frac{1}{6}, & x \in\left[\frac{2}{3}, 1\right],\end{cases} \\
T(x)= \begin{cases}-\frac{1}{4}, & x \in\left[-1, \frac{2}{3}\right), \\
-\frac{1}{7}, & x \in\left[\frac{2}{3}, 1\right],\end{cases} \\
R(x)= \begin{cases}-\frac{1}{4}, & x \in\left[-1, \frac{2}{3}\right), \\
-\frac{1}{5}, & x \in\left[\frac{2}{3}, 1\right],\end{cases} \\
f(x)= \begin{cases}x, & x \in\left[-1, \frac{2}{3}\right), \\
\frac{2}{3}, & x \in\left[\frac{2}{3}, 1\right] .\end{cases}
\end{gathered}
$$

Note that $F, T, R$, and $f$ satisfy

$$
2 G_{k}(F x, T y, R z) \leq \frac{1}{5} M_{k}(x, y, z)+L N_{k}(x, y, z),
$$

for all $x, y, z \in X$ and $L \geq 0$. In addition $f(X)=\left[-1, \frac{2}{3}\right]$ is a $G_{b}$-complete subspace of $X, F(X) \cup T(X) \cup R(X) \subset f(X)$, and the pairs $(f, F),(f, T)$, and $(f, R)$ are weakly compatible. All the conditions of Theorem 2 are thus verified. As a consequence $-\frac{1}{4}$ is the unique point of coincidence and the unique common fixed point for all of the mappings $F, T, R$, and $f$.

The following result derives from Theorem 2 .

Corollary 2. Let $(X, G)$ be a cone $G$-metric space relative to a normal constant $k \geq 1$. Suppose that the mappings $F, T, R, f: X \rightarrow X$ satisfy

$$
G_{k}(F x, T y, R z) \leq \lambda M_{k}(x, y, z)+L N_{k}(x, y, z),
$$

for all $x, y, z \in X, \lambda \in\left[0, \frac{1}{2 k}\right)$ and $L \geq 0$. If $F(X) \cup T(X) \cup R(X) \subset f(X)$ and $f(X)$ is a $G$-complete subspace of $X$, then $F, T, R$, and $f$ have a unique point of coincidence in $X$. If further the pairs $(f, F),(f, T)$ and $(f, R)$ are weakly compatible, then $F, T, R$, and $f$ have a unique common fixed point.

\section{Remark 1.}

(1) Let $F=R=S$ and $f=I$ in Theorem 1. Then Theorem 1 improves [3, Theorem 3.2].

(2) Corollary 1 with $F=R=S$ extends [9, Theorem 2.1]. 
(3) If in Corollary 1, we set $F=R=S$ and $f=I$, then we obtain an extension of [11, Theorem 2.7].

(4) $T=R$ and $z=y$ in Theorem 2 leads to a generalization of [16, Theorem 2.1] with $M=T$.

(5) Corollary 2 with $F=R=S, f=I$, and $z=y$ is an extension of [2, Theorem 2.1, Theorem 2.5, Theorem 2.8].

\section{Acknowledgements}

The authors are grateful to the Direction Générale de la Recherche Scientifique et de Développement Technologique in Algeria for supporting this work. The authors thank the anonymous referees for their careful reading of the original manuscript.

\section{References}

[1] A.E. Al-Mazrooei; J. Ahmad. Fixed point results for multivalued mappings in $g_{b}$-cone metric spaces. J. Nonlinear Sci. Appl., 10(9):4866-4875, 2017.

[2] Z. Mustafa; H. Obiedat; F. Awawdeh. Some fixed point theorem for mapping on complete $g$-metric spaces. Fixed Point Theory Appl., Art. ID 189870:12 pp., 2008.

[3] M. Ughade; R.D. Daheriya. Fixed point results for contraction mappings in $g_{b}$-cone metric spaces. Gazi Univ. J. Sci., 28:659-67, 2015.

[4] R.P. Agarwal; E. Karapınar; D. O’Regan; A.F. Roldán-López de Hierro. Fixed point theory in metric type spaces. Springer, Cham., page 385 pp., 2015.

[5] D. Dorić. Common fixed point theorems for generalized multivalued contractions on cone metric spaces over a non-normal solid cone. Fixed Point Theory Appl., 159:12 pp., 2014.

[6] Sh. Rezapour; R. Hamlbarani. Some notes on the paper: "cone metric spaces and fixed point theorems of contractive mappings" [j. math. anal. appl. 332 (2007), no. 2, 1468-1476; mr2324351] by l.-g. huang and x. zhang. J. Math. Anal. Appl., 345(2):719$724,2008$.

[7] M. Abbas; G. Jungck. Common fixed point results for noncommuting mappings without continuity in cone metric spaces. J. Math. Anal. Appl., 341(1):416-420, 2008 .

[8] R.D. Daheriya; M. Ughade; M. Likhitker. A common coupled fixed point theorem with contractive type condition in $g_{b}$-cone metric space. Asian Research Journal of Mathematics., 1(3):1-18, 2016. 
[9] I. Beg; M. Abbas; T. Nazir. Common fixed point results in $g$-cone metric spaces. $J$. Adv. Res. Pure Math., 2(4):94-109, 2010.

[10] I. Beg; M. Abbas; T. Nazir. Generalized cone metric spaces. J. Nonlinear Sci. Appl., $3(1): 21-31,2010$.

[11] I. Beg; M. Abbas; T. Nazir. Fixed point results in generalized cone metric spaces. Acta Univ. Apulensis Math. Inform., 28:215-232, 2011.

[12] S. Aleksić; Z. Kadelburg; Z. D. Mitrović; S. Radenović. A new survey: Cone metric spaces. J. Int. Math. Virtual Inst., 9:93-121, 2019.

[13] S. Janković; Z. Kadelburg; S. Radenović. On cone metric spaces: a survey. Nonlinear Anal., 74(7):2591-2601, 2011.

[14] M. Abbas; B.E. Rhoades. Fixed and periodic point results in cone metric spaces. Appl. Math. Lett., 22(4):511-515, 2009.

[15] A. Aghajani; M. Abbas; J.R. Roshan. Common fixed point of generalized weak contractive mappings in partially ordered $g_{b}$-metric spaces. Filomat., 28(6):10871101, 2014.

[16] M. Koierng Meitei; R. Yumnam; R.S. Verma. Some common fixed point theorems for two pairs of weak compatible mappings of type (a) in $g_{b}$-metric space. American Journal of Applied Mathematics and Statistics., 6(4):135-140, 2018.

[17] D. Wardowski. Endpoints and fixed points of set-valued contractions in cone metric spaces. Nonlinear Anal., 71(1-2):512-516, 2009.

[18] L.-G. Huang; X. Zhang. Cone metric spaces and fixed point theorems of contractive mappings. J. Math. Anal. Appl., 332(2):1468-1476, 2007. 